\title{
Substructure of bovine casein micelles by small-angle X-ray and neutron scattering
}

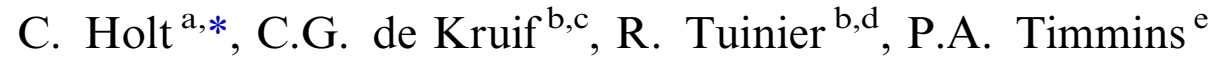 \\ ${ }^{a}$ Hannah Research Institute, Ayr, Scotland, UK \\ ${ }^{\mathrm{b}}$ Van't Hoff Laboratory for Physical and Colloid Chemistry, Debye Institute, University of Utrecht, Padualaan 8, 3584 CH Utrecht, The \\ Netherlands \\ ${ }^{\mathrm{c}}$ NIZO food research, P.O. Box 206710 BA Ede, The Netherlands \\ ${ }^{\mathrm{d}}$ Institut Für Festkörperforschung, Wieche Materie, Forschungszentrum 54425, Julich, Germany \\ e Institut Max Von Laue - Paul Langevin, Grenoble, France \\ Received 30 April 2002; accepted 9 August 2002
}

\begin{abstract}
The casein micelles of cow's milk are polydisperse, more-or-less spherical, protein particles of up to several hundred nanometer in size, containing about $7 \%$ by dry weight of calcium phosphate. Small-angle neutron scattering with contrast variation and small-angle X-ray scattering were used in critical tests of models of casein micelle substructure. An inflexion in the neutron scattering curve near $Q=0.35 \mathrm{~nm}^{-1}$ was observed in heavy water which became a more pronounced subsidiary maximum at the match point of the protein. In water-rich buffers, where the contrast between protein and calcium phosphate is small, the inflexion was less apparent. The position of the inflexion and its variation in shape and relative importance with contrast matching are explained poorly, if at all, by the submicelle models of casein micelle substructure. However, the observations are explained by a model in which a relatively uniform protein matrix contains a disordered array of calcium phosphate ion clusters. A notable achievement of the model is the prediction of the position of the subsidiary maximum from independent measurements of the intrinsic viscosity of micelles, their mass fraction of calcium phosphate and the mass of the core of a calcium phosphate nanocluster.
\end{abstract}

(C) 2002 Elsevier Science B.V. All rights reserved.

Keywords: Small-angle; X-ray scattering; Calcium phosphate; Casein micelle

\section{Introduction}

In bovine milk, the casein micelles are large, polydisperse and heterogeneous protein structures,

\footnotetext{
* Corresponding author.

E-mail address: holtc@hri.sari.ac.uk (C. Holt).
}

the physiological functions of which probably include the safe transport of an insoluble calcium salt from mother to young [1]. The number distribution of radii is approximately $\log -$ normal in shape with a maximum at a radius of $\sim 100 \mathrm{~nm}$ [2]. The micelles are chemically heterogeneous because the proteins are held together, for the 
most part, by a small mass fraction, about 0.07 , of calcium phosphate.

The substructure of casein micelles has been studied by small-angle X-ray [3] and neutron [4-6] scattering (SAXS and SANS, respectively). An inflexion point is seen by SANS in heavy water at about $Q=0.35 \mathrm{~nm}^{-1}$. The wave vector $Q$ is defined as $4 \pi \sin (\theta / 2) / \lambda_{\mathrm{o}}$, where $\theta$ is the angle at which the scattered intensity is detected and $\lambda_{\mathrm{o}}$ is the X-ray or neutron wavelength. All the SAXS and SANS studies published to date have assumed that the inflexion is due to scattering by submicelles. Submicellar models of casein micelle substructure have been proposed in a wide variety of forms over the last 35 years (reviewed by Rollema [7]). They have included models in which the submicelles are of constant, or variable, size, composition or internal structure. In some models the submicelles are held together by calcium phosphate, in others, the calcium phosphate does not feature and in the most recent proposal [8], the calcium phosphate is placed within the submicelles. Other workers have not accepted the subunit hypothesis [1,9-15] and have proposed that the substructure might be due to the micellar calcium phosphate.

When a solution containing the hydrophilic $\beta$ casein $\mathrm{N}$-terminal tryptic phosphopeptide, $\beta-\mathrm{CN}$ 4P (f1-25), and milk salts at their milk concentrations was gently brought up in $\mathrm{pH}$ from 5.5 to 6.7 by hydrolysis of urea with the enzyme urease, particles called calcium phosphate nanoclusters formed spontaneously [15]. These comprised a core of calcium phosphate and a shell of the phosphopeptide. The core calcium phosphate was found to be amorphous and was closely similar in its composition and solubility to the natural casein micellar calcium phosphate.

In this paper, we largely confirm and extend earlier observations of SANS by casein micelles using contrast variation, and interpret the results using a model for the micelle substructure in which a more-or-less homogeneous protein matrix contains a disordered array of calcium phosphate particles with the same mass as the core mass of calcium phosphate nanoclusters prepared in the laboratory.

\section{Theory}

In a SANS experiment, the normalised scattered intensity $I(Q)$ is measured. For a dispersion of isotropic, monodisperse particles, $I(Q)$ is related to the inter-particle structure factor, $S(Q)$ and the particle scattering factor $P(Q)$ by [16-18]:

$I(Q) \propto \phi P(Q) S(Q)$,

where $\phi$ is the volume fraction of colloidal particles. The proportionality constant depends on the difference between the scattering length densities of the particle and the solvent. For homogeneous spherical colloids of radius $R$, the particle scattering factor is:

$$
\begin{aligned}
& P_{\text {sphere }}(Q, R) \\
& \quad=\left\{3\left(\frac{\sin (Q R)-Q R \cos (Q R)}{(Q R)^{3}}\right)\right\}^{2} .
\end{aligned}
$$

Here we assume that casein micelles are essentially polydisperse spheres that are homogeneous apart from the presence of the calcium phosphate. Both polydispersity and the statistical nature of a structure act to smooth out the sharp features in the particle scattering factor of the homogeneous sphere. Eq. (2) is approximated by $P(Q)=\exp (-$ $\left.Q^{2} R_{\mathrm{g}}^{2} / 3\right)$ for $Q R_{\mathrm{g}}<1$. Hence, a so-called Guinierplot of $\log I$ vs. $Q^{2}$ gives the radius of gyration, $R_{\mathrm{g}}$, from the slope.

\subsection{Subunit model of the substructure}

For a spherical particle formed from a large number, $N_{\text {sub }}$, of identical subunits of radius $r_{\text {sub }} \ll R$, the particle scattering factor can be written as:

$$
\begin{aligned}
P(Q, R)= & P_{\text {sphere }}(Q, R) \\
& \times\left[N_{\text {sub }}+N_{\text {sub }}^{2} P_{\text {sphere }}\left(Q, r_{\text {sub }}\right)\right] .
\end{aligned}
$$

Since $r_{\text {sub }} \ll R$, the effect of polydispersity in subunit size and particle size on $I(Q)$ can be treated independently because they affect different ranges of $Q$. The subunit structure introduces a correlation length of $2 r_{\text {sub }}$ into the scattering contrast variation within the particle to produce a shoulder or subsidiary maximum in $I(Q)$, at or about $Q=\pi / r_{\text {sub }}$. In fact, the polydispersity in $r_{\text {sub }}$ 
and packing irregularities produce a strong smearing of the fine structure in the particle scattering factor. Since, according to this model, the origin of the correlation length lies in the geometry of the cluster of subunits, it should be seen at all contrasts except for the match point of the protein.

\subsection{Calcium phosphate nanocluster model of the substructure}

Now consider a homogeneous protein sphere containing $N_{\mathrm{CaP}}$ calcium phosphate clusters, each of mass $m$. The calcium phosphate nanoclusters are assumed to form a disordered array with a characteristic correlation length $\lambda$. A regular lattice would give rise to diffraction and Bragg peaks. The correlation length corresponds roughly to the first maximum in the pair correlation function of the disordered material, the Fourier transform of which gives rise to the observed scattering. In a casein micelle of mass $M$, volume $V$, and with a mass fraction of calcium phosphate, $w$, there are $N_{\mathrm{CaP}}$ nanoclusters:

$N_{\mathrm{CaP}} \approx \frac{V}{\lambda^{3}}=\frac{w M}{m}$.

It is convenient to use hydrodynamic measurements of casein micelle size such as the intrinsic viscosity, $[\eta]=2.5 V_{\mathrm{h}}$, where $V_{\mathrm{h}}$ is the hydrodynamic volume. Assuming the calcium phosphate is only in the non-draining inner region of the micelle, the correlation length becomes:

$\lambda^{3}=\frac{2 m[\eta]}{5 w N_{\mathrm{A}}}$,

where $N_{\mathrm{A}}$ is Avogadro's number.

In contrast variation studies of proteins, the contrast between protein and solvent and between calcium phosphate and solvent is commonly varied by using mixtures of normal and heavy water. Allowing for exchange, the scattering length densities of $\beta$-casein 4P (f1-25) and the acidic amorphous calcium phosphate in calcium phosphate nanoclusters [15], $\rho_{\text {prot }}$ and $\rho_{\mathrm{CaP}}$, respectively, are given by:

$\rho_{\text {prot }}=1.901+1.011 \beta$
$\rho_{\mathrm{CaP}}=2.019+4.043 \beta$,

where $\beta$ is the volume fraction of heavy water. The predicted match points for the $\alpha_{\mathrm{s}^{-}}, \alpha_{\mathrm{s} 2^{-}}, \beta$ - and $\kappa-$ caseins occur at $\beta=0.41,0.42,0.38$ and 0.41 , respectively [5]. Additionally, Eq. (6) shows that near $\beta=0$, the protein and calcium phosphate have almost the same contrast.

$I(Q) \propto P(Q, R) \sim P_{\text {sphere }}(Q, R)$.

Near $\beta=0.4$, the match point for whole casein, we have:

$I(Q) \propto P(Q, a) S(Q, \lambda)$,

where $a$ is the radius of the calcium phosphate particles, $P(Q, a)$ is the particle scattering factor of the ion cluster and $S(Q, \lambda)$ is the inter-particle scattering function for the nanocluster array within the micelle. In heavy water, the scattering length density of the calcium phosphate is twice that of the protein so it is an important source of coherently scattered neutrons at $Q R>10$, in spite of its relatively small mass fraction.

The intrinsic viscosity of the casein micelle is typically $10 \mathrm{~cm}^{3} \mathrm{~g}^{-1}$ [19], the mass fraction of calcium phosphate is about $0.07[1,20]$ and the relevant properties of calcium phosphate nanoclusters prepared from $\beta$-casein phosphopeptide $4 \mathrm{P}$ (f1-25) are [15] $m=61 \mathrm{kDa}$, the core mass, and $a=2.3 \mathrm{~nm}$, the core radius. Thus, if the micellar calcium phosphate is the same as the core of calcium phosphate nanoclusters, $\lambda=18 \mathrm{~nm}$ and, since $Q^{\prime} a=0.8, P_{\text {sphere }}\left(Q^{\prime}, a\right)=0.77$ and the characteristic feature arising from the inter-particle scattering of the calcium phosphate is expected to occur at $Q=0.35 \mathrm{~nm}^{-1}$. A casein micelle with the number average radius in the size distribution of $108 \mathrm{~nm}$ [2] has a mass of $7.2 \times 10^{8} \mathrm{Da}$ and, according to the model, contains 830 calcium phosphate ion clusters.

\section{Experimental}

\subsection{Materials}

Casein micelles from fresh skim milk were pelleted by ultracentrifugation $(25000 \times g$ for 30 
$\min$ at $20{ }^{\circ} \mathrm{C}$ ) before resuspension in a buffer designed to be saturated with respect to micellar calcium phosphate. The composition and preparation of such buffers has been described previously $[10,14,15]$ where they were used to dilute caseincalcium phosphate complexes, while preserving their integrity. The standard resuspension buffer had a free calcium ion concentration of $2 \mathrm{mM}$, ionic strength of $80 \mathrm{mM}$ and $\mathrm{pH}$ of 6.7. In experiments where ethanol was used to sharpen the feature at about $0.35 \mathrm{~nm}^{-1}$, the ethanol was added slowly to the buffer to a final concentration of 5,10 or $15 \% \mathrm{v} / \mathrm{v}$. Identical buffers were prepared in normal and heavy water and mixed to give the required $\beta$ value. After resuspension, to give a stock casein concentration of about $10 \mathrm{mg}$ protein $\mathrm{ml}^{-1}$, samples were dialysed over $3 \mathrm{~d}$ against 3 changes of the same buffer at a volume ratio of $1: 3$. The precise value of $\beta$ in the sample and dialysis buffer was determined by a neutron transmission experiment using pure water and heavy water as standards and was usually lower than the nominal value by about $0.02-0.04$.

\subsection{Methods}

\subsubsection{Small-angle $X$-ray scattering}

This was carried out on station 2.1 of the CCLRC Daresbury Laboratory at camera lengths of 2.3 or $6.25 \mathrm{~m}$. Measurements were made at protein concentrations of approximately 10,5 or 1 $\mathrm{mg} \mathrm{ml}^{-1}$. Other experimental details were described previously [15].

\subsubsection{Small-angle neutron scattering}

The SANS experiments were performed at the Institut Max Von Laue-Paul Langevin in Grenoble (France) on the D11 and D22 spectrometers with camera lengths of 3,5 or $18 \mathrm{~m}$. Hellma QS quartz cells were used with a sample path length of $2 \mathrm{~mm}(0.6 \leq \beta \leq 1)$ or $1 \mathrm{~mm}$. The mean wavelength of the emitted neutrons was $1.0 \mathrm{~nm}$ with a width at half height of $9 \%$. A circulating water bath was used to control the sample temperature to \pm $0.5{ }^{\circ} \mathrm{C}$ at $25{ }^{\circ} \mathrm{C}$, unless otherwise stated. Measurements were also made at protein concentrations of 5 and $20 \mathrm{mg} \mathrm{ml}^{-1}$ to verify that near the point of inflexion, $Q=0.35 \mathrm{~nm}^{-1}, S(Q, R)$ does not affect the shape of the scattering curve in this concentration range. Samples were in dialysis equilibrium with the buffer and the coherent scattering from the solute was obtained by subtraction of the buffer scattering from the sample.

\section{Results}

\subsection{Small-angle X-ray scattering}

The stock solution was diluted until there were no concentration-dependent features in the SAXS curve of $\log I$ vs. $Q$. This occurred at or below 5 $\mathrm{mg}$ protein $\mathrm{ml}^{-1}$. As was found previously [3], there was no distinct inflexion point in the SAXS of resuspended casein micelles near $Q=0.35$ $\mathrm{nm}^{-1}$. In this study, therefore, the SAXS was used to help define optimal conditions for the SANS. It was found that an inflexion could be induced by addition of ethanol and it became an increasingly prominent shoulder as the concentration increased to $15 \%$ (Fig. 1). At $20 \%$ the micelles were found to be unstable and slowly aggregated so the optimal concentration for future work was chosen to be $15 \%$. The $\mathrm{pH}$ of the resuspension buffer was varied from 6.0 to 8.0 in steps of $0.5 \mathrm{pH}$ units in the presence of $15 \%$ ethanol and the SAXS showed that the most prominent inflexion was at $\mathrm{pH}$ 7.0. At $\mathrm{pH} 6$ the micellar calcium phosphate had largely dissolved but the micelles remained intact because of the reduced charge repulsion [19]. Above $\mathrm{pH} \mathrm{7,} \mathrm{the} \mathrm{micelle} \mathrm{suspension} \mathrm{became}$ progressively less turbid due to partial dissociation of the particles even though the micellar calcium phosphate is expected to increase. The effect of replacing water with heavy water was investigated with another system composed of a mixture of $\beta$ and $\kappa$-caseins together with calcium phosphate which can form particles small enough for a Guinier analysis by SAXS. The larger particles, formed at low ratios of $\kappa$ - to $\beta$-caseins showed a distinct secondary maximum in the SAXS but this became less prominent with the smaller particles. No dependence of $R_{\mathrm{g}}$ on $\beta$ was found in the range $0 \leq \beta \leq 0.96$ using a stock mixture prepared from $8 \mathrm{mg} \beta$-casein $+10 \mathrm{mg} \kappa$-casein $\mathrm{ml}^{-1}(\mathrm{CH}$, unpublished observations). This result was consid- 


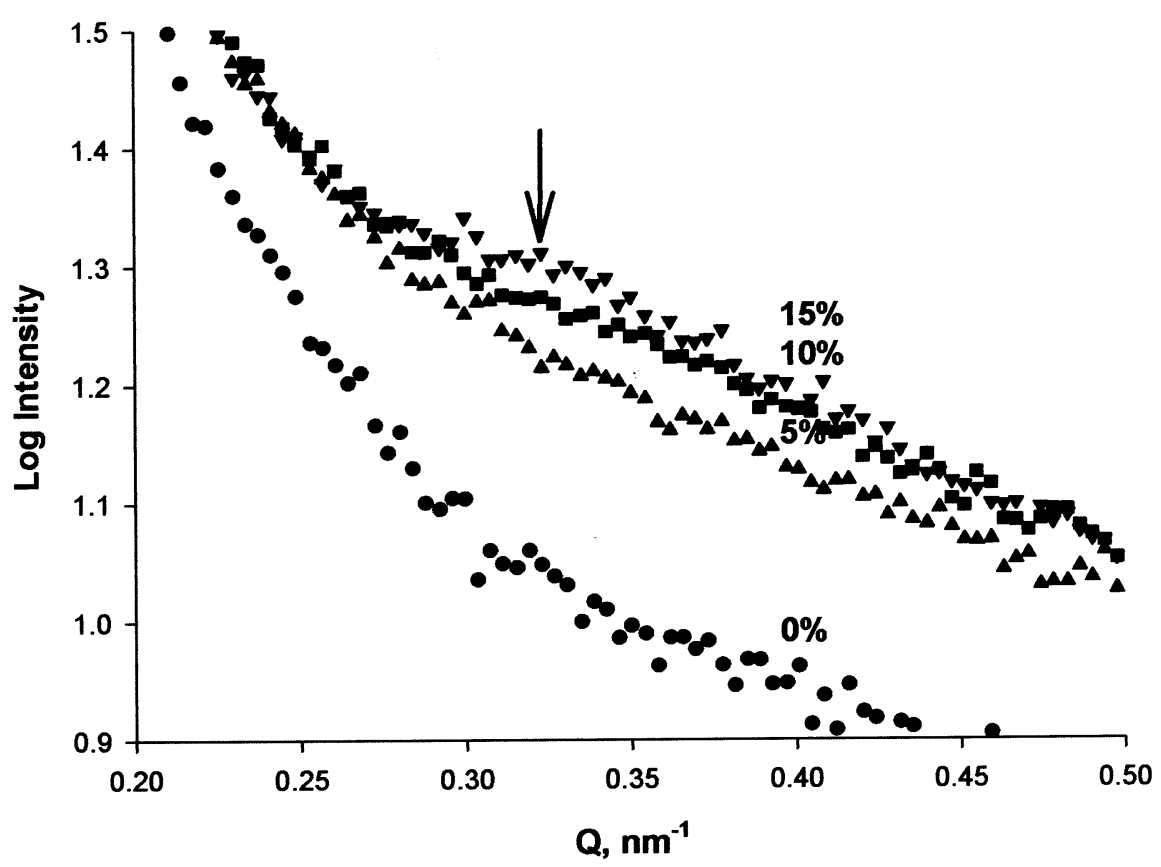

Fig. 1. SAXS of resuspended casein micelles in aqueous buffers containing 0, 5, 10 and 15\% ethanol.

ered good evidence that the size and substructure of the native casein micelle was unlikely to be perturbed by the exchange of normal for heavy water in a contrast variation study by SANS.

\subsection{Small-angle neutron scattering}

The initial experiment was the determination of the SANS of resuspended casein micelles at nominal $\beta$ values of $0,0.2,0.6,0.7,0.8,0.9$ and 1. There was no added ethanol in this series. As shown in Fig. 2(a), a shoulder near $Q=0.35 \mathrm{~nm}^{-1}$ was found in the heavy water-rich solutions and even appeared to become more prominent nearer the match point of the protein. However, in the aqueous solutions $(\beta=0.2$ or 0$)$, no shoulder was found. The effect of ethanol in sharpening the inflexion to a distinct maximum was also observed in heavy water by SANS (result not shown). In a second experiment, measurements were made closer to the match point at nominal $\beta$ values of 0.42 and 0.5 , allowing for the $15 \%$ ethanol added to the buffers. In spite of the lower intensity of coherently scattered neutrons, the feature appeared as a distinct maximum at $Q=0.35 \mathrm{~nm}^{-1}$ (Fig. 3). No other features appeared in the SANS at lower $Q$ in measurements made with the $\beta=0.5$ solution at the longer camera length of $18 \mathrm{~m}$, as would be expected of a regular lattice.

In the final experiment, the SANS was measured in a buffer of $\mathrm{pH} 7.0$ containing $15 \%$ ethanol at a nominal $\beta$ value of 0.6 . An initial measurement was made at $5{ }^{\circ} \mathrm{C}$, the solution was then raised in temperature to $60{ }^{\circ} \mathrm{C}$ and a second measurement of the SANS made. The sample was then cooled to $5{ }^{\circ} \mathrm{C}$ and the SANS re-recorded. The measurement time was $1 \mathrm{~h}$ and 20-40 min was allowed for equilibration before each determination. Results are shown in Fig. 4 and comparison with Fig. 2 shows that the shoulder seen without the ethanol was again converted into a distinct maximum at about $Q=0.35 \mathrm{~nm}^{-1}$ at $5{ }^{\circ} \mathrm{C}$. On heating to $60{ }^{\circ} \mathrm{C}$ the maximum became less prominent. Fig. 4 shows, however that the SANS was almost completely recovered after the heat treatment cycle, demonstrating the reversibility of the change. 

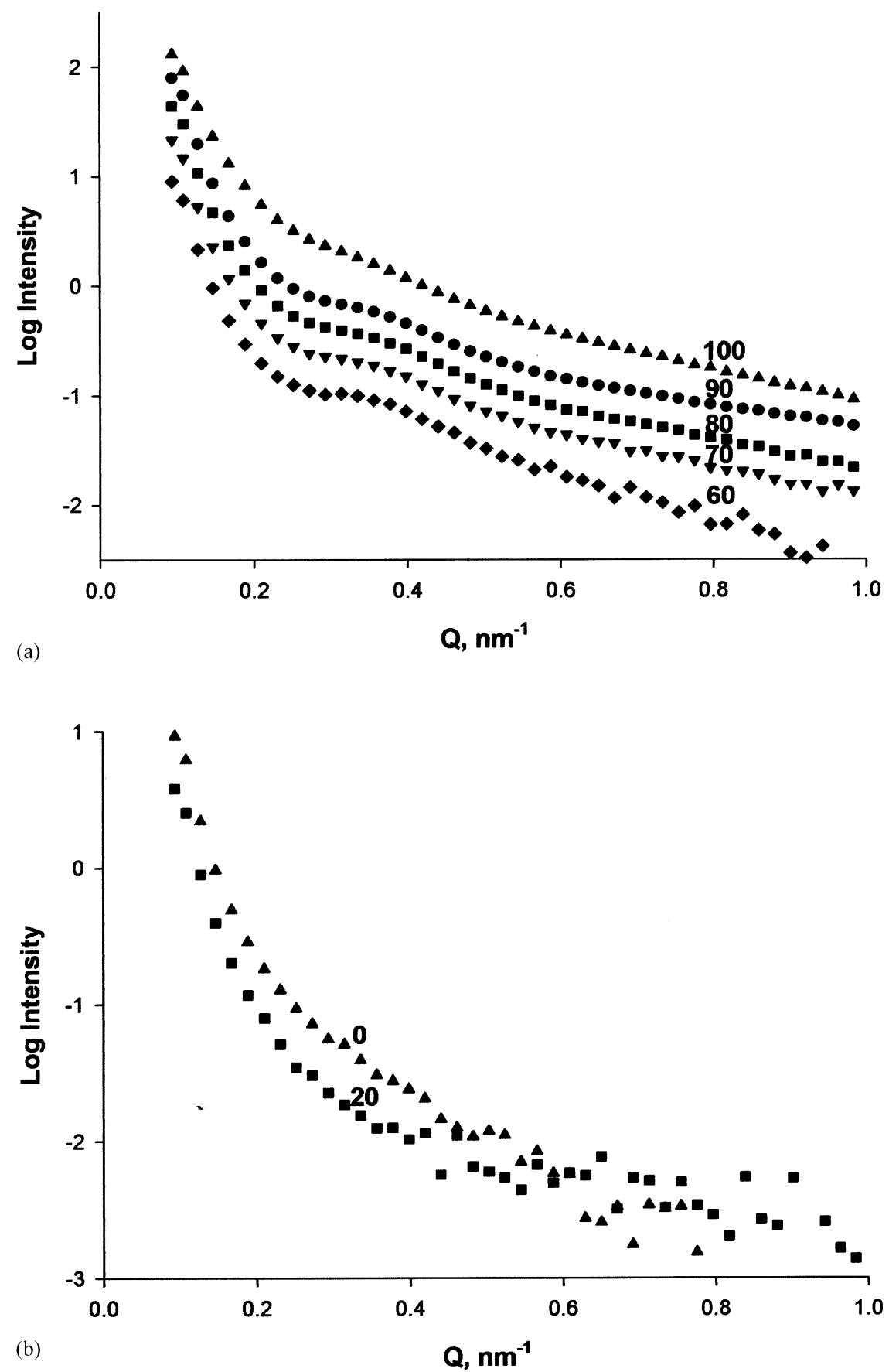

Fig. 2. (a) SANS of resuspended casein micelles above the match point of the protein at $\beta=0.6,0.7,0.8,0.9$ and 1 . None of the solutions contained ethanol. (b) SANS of resuspended casein micelles at $\beta=0$ and 0.2 , near the match point of protein to the calcium phosphate. 


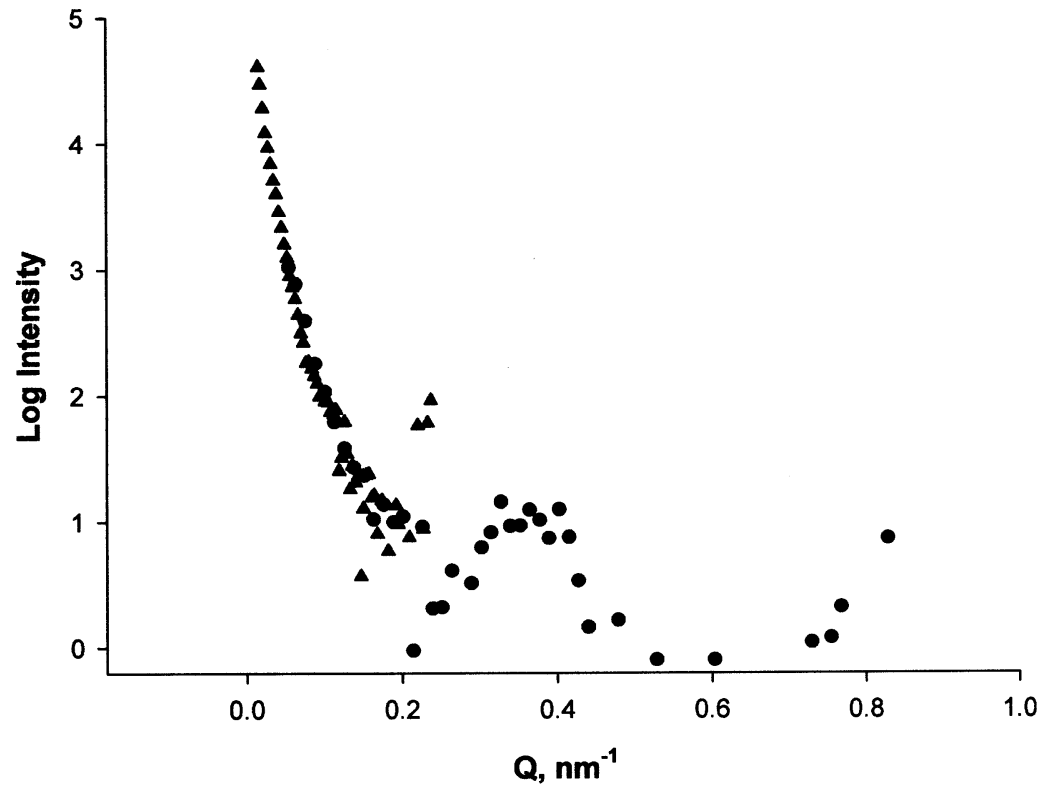

Fig. 3. SANS of resuspended casein micelles with $\beta=0.5$ and camera lengths of 18 and $5 \mathrm{~m}$. A least squares minimisation routine was used to overlap the two sets of data.

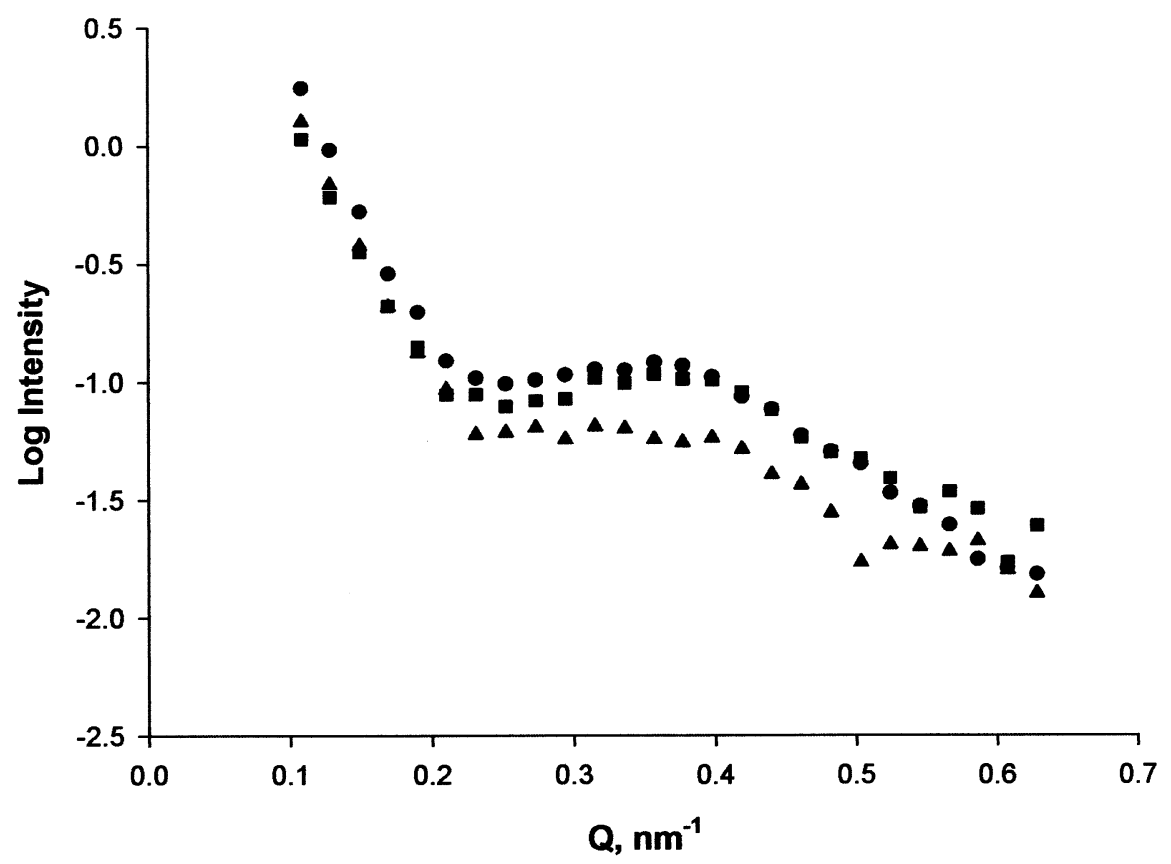

Fig. 4. SANS of resuspended casein micelles in a buffer containing $15 \%$ ethanol at $5{ }^{\circ} \mathrm{C}$ (before heating), $\mathbf{0}$; $60 \quad{ }^{\circ} \mathrm{C}$, $\Delta$; and $5 \quad{ }^{\circ} \mathrm{C}$ (after heating), 


\section{Discussion}

Early measurements of SANS by casein micelles $[4,5]$ were interpreted in terms of small, hexagonally packed, clusters of homogeneous subunits with $r_{\text {sub }}=8.4 \mathrm{~nm}$. One reason for choosing a subunit model for the interpretation was that a number of studies by electron microscopy had revealed casein-like particles of $5-25 \mathrm{~nm}$ in the Golgi region of mammary secretory cells, as well as casein micelle-sized particles and it was presumed that the smaller particles assembled into micelles [20]. The second reason was that a number of reports were published in which particles of the size of subunits were isolated by dissociation procedures [20]. Stothart [5], assuming a tricalcium phosphate stoichiometry, calculated that the micellar calcium phosphate was contrast-matched at $\beta=0.74$, where the shoulder at $Q=0.35 \mathrm{~nm}^{-1}$ is still evident, and concluded that the shoulder was not caused by scattering from the calcium phosphate. We argue against this conclusion on the grounds that a correlation length arises from the variation in scattering length density within the particle and that 'holes' of zero contrast with the solvent nevertheless give rise to an inter-'hole' interference effect. Stothart noted [5] that there was not just a shoulder but a distinct peak at $\beta=$ 0.38 , close to the calculated match point for the protein, but explained this as arising from local variations in the scattering length density of the protein about the mean value. We accept that there are variations in contrast at the match point for protein but ascribe them mainly to the calcium phosphate with its distinctly different scattering length density. Stothart and Cebula [4,5] did not report measurements in water-rich solvents, where we found that the shoulder or peak at $Q=0.35$ $\mathrm{nm}^{-1}$ was absent or weak. In water-rich solvents, the scattering length densities of the protein and calcium phosphate are almost the same so the casein micelles should scatter neutrons more like homogeneous particles. Hansen et al. [6] also worked with an aqueous buffer and found that the inflexion point was still present in the $\beta=0$ solution, although less prominent and somewhat shifted in position relative to the SANS curve obtained at $\beta=1$. Like Stothart [4,5], Hansen et al. [6] used a protein subunit model to describe the scattering. In order to fit the SANS curves as a function of the scattering contrast, the subunits were allowed to be polydisperse with a Gaussian distribution. They were found to have a mean radius that decreased from $6.7 \pm 3.7 \mathrm{~nm}$ at $\beta=1$ to $4.5 \pm 2.2 \mathrm{~nm}$ at $\beta=0$. The variation in contrast within the micelle was noted to be very large by protein standards and the inside of the putative subunits were not contrast matched at the match point of the protein.

The inflexion point, in our analysis, is ascribed to inter-particle interference arising from the scattering of calcium phosphate particles. We have not quantified the effect of the cross interaction between scattering from protein and calcium phosphate, as would be expected to be significant near $\beta=1$, but note that there is no reason to expect this effect to give rise to a characteristic correlation length and therefore provide an alternative explanation for the inflexion.

The subunit model of casein micelles has been criticised for a number of reasons. First, it is not established that the putative submicelles seen by electron microscopy persist in the casein micelle or indeed that the micelle is built from these units. Moreover, when casein micelles are carefully dissociated in a variety of ways to probe the forces holding the micelle together, particles of every possible size up to that of the original micelles can result [10]. The older electron microscopical evidence of casein micelle substructure has also been put into question by a more recent careful study of the effects of various preparation procedures. There were no inhomogeneities larger than a few nanometer in casein micelle samples that were prepared by methods that did not introduce artefacts [21]. In this respect, the inhomogeneities are comparable to those seen much earlier by transmission electron microscopy in unstained thin sections [12].

Our position is that submicelles are an unnecessary hypothesis; the substructure of casein micelles can be explained by the presence of micellar calcium phosphate. The scale of the substructure, most clearly seen in the SANS at the match point of the protein, is correctly predicted by Eq. (5) from a model of micellar calcium phosphate based 
on completely independent experiments. These are the intrinsic viscosity of casein micelles, the weight fraction of calcium phosphate in casein micelles and the core mass of calcium phosphate nanoclusters prepared in the laboratory.

The fact that the shoulder or peak near $Q=0.35$ $\mathrm{nm}^{-1}$ is much more prominent at the match point of the protein than at any other solvent contrast, a consistent result in all the previous studies [4-6], and this one, is strong evidence that the feature is due to interparticle interference by the calcium phosphate (Eq. (5)). When the contrast of the calcium phosphate approaches that of the protein in water-rich solvents, the shoulder or peak near $Q=0.35 \mathrm{~nm}^{-1}$ becomes weakened [6] or, as reported here, may be essentially absent. This result is difficult to reconcile with a protein subunit model (Eq. (3)) but is exactly as expected of a calcium phosphate nanocluster model (Eq. (8)). The effect of ethanol addition and heating are seen as having opposite effects on the dispersion in the correlation length, $\lambda$, to cause, respectively, sharpening or smoothing of the inter-particle scattering function arising from the disordered array of micellar calcium phosphate clusters. Although concentrations of ethanol comparable to those used here have been shown to cause the contraction of the outermost region of the casein micelle [22], the so-called hairy layer that confers steric stability on the particles, it appears from this work that it affects also the inner substructure. Hansen et al. [6] confirmed that the substructure was preserved on renneting and we have evidence from SAXS that the prominence of the shoulder is reduced or eliminated by acidification to $\mathrm{pH} 6.0$ over the 2-3 days of sample preparation, consistent with a slow readjustment of the internal structure to the loss of the micellar calcium phosphate. The prominence of the shoulder is also reduced at alkaline $\mathrm{pH}$ which may be caused by the reduction of particle size at the higher $\mathrm{pH}$.

\section{Acknowledgements}

We are indebted to Elaine Little (HRI) for experimental assistance in preparing the casein micelle samples and buffers. Dr G. Grossmann
(CLRC Daresbury Laboratory) is thanked for his help with the SAXS measurements. The work was financed primarily by the Scottish Executive Environmental and Rural Affairs Department.

\section{References}

[1] C.G. de Kruif, C. Holt, Casein micelle structure, functions and interactions, in: P.F. Fox, P.L.H. McSweeney (Eds.), Advanced Dairy Chemistry Proteins, vol. 1, Kluwer Academic, Plenum, New York, 2002, pp. 233-276.

[2] C.G. de Kruif, Supra-aggregates of casein micelles as a prelude to coagulation, Journal of Dairy Science 81 (1998) 3019-3028.

[3] H. Pessen, T.F. Kumosinski, H.M. Farrell, Small-angle Xray scattering investigation of the micellar and submicellar forms of bovine casein, Journal of Dairy Research 56 (1989) 443-451.

[4] P.H. Stothart, D.J. Cebula, Small-angle neutron scattering study of bovine casein micelles and sub-micelles, Journal of Molecular Biology 160 (1982) 391-395.

[5] P.H. Stothart, Subunit structure of casein micelles from small-angle neutron scattering, Journal of Molecular Biology 208 (1989) 635-638.

[6] S. Hansen, R. Bauer, S.B. Lomholt, K. Bruun Qvist, J.S. Pedersen, K. Mortensen, Structure of casein micelles studied by small-angle neutron scattering, European Biophysics Journal 24 (1996) 143-147.

[7] H.S. Rollema, Casein association and micelle formation, in: P.F. Fox (Ed.), Advanced Dairy Chemistry, vol. 1, Elsevier Applied Science, Barking, Essex, UK, 1992, pp. $111-140$.

[8] P. Walstra, Casein sub-micelles: do they exist?, International Dairy Journal 9 (1999) 189-192.

[9] C. Holt, Structure and stability of bovine casein micelles, Advances in Protein Chemistry 43 (1992) 63-151.

[10] C. Holt, Casein micelle substructure and calcium phosphate interactions studied by sephacryl column chromatography, Journal of Dairy Science 81 (1998) 29943003.

[11] D. Rose, J.R. Colvin, Internal structure of casein micelles from bovine milk, Journal of Dairy Science 49 (1966) 351355.

[12] A.-M. Knoop, E. Knoop, A. Wiechen, Sub-structure of synthetic casein micelles, Journal of Dairy Research 46 (1979) 347-350.

[13] D.S. Horne, Casein interactions: casting light on the black boxes, the structure in dairy products, International Dairy Journal 8 (1998) 171-177.

[14] C. Holt, D.T. Davies, A.J.R. Law, The effects of colloidal calcium phosphate content and milk serum free calcium ion concentration on the dissociation of bovine casein micelles, Journal of Dairy Research 53 (1986) $557-572$. 
[15] C. Holt, P.A. Timmins, N. Errington, J. Leaver, A core-shell model of calcium phosphate nanoclusters derived from sedimentation equilibrium and small angle X-ray and neutron scattering measurements, European Journal of Biochemistry 252 (1998) $73-78$.

[16] B. Jacrot, The study of biological structures by neutron scattering from solution, Reports on the Progress of Physics 39 (1976) 911-953.

[17] L.A. Feigin, D.I. Svergun, Structural Analysis by SmallAngle X-ray and Neutron Scattering, Plenum, NewYork, 1987.

[18] M. Kerker, Scattering of Light and Other Electromagnetic Radiation, Academic Press, New York, 1969.
[19] T.J.M. Jeurnink, C.G. de Kruif, Changes in milk on heating: viscosity measurements, Journal of Dairy Research 60 (1993) 139-150.

[20] D.G. Schmidt, Association of caseins and casein micelle structure, in: P.F. Fox (Ed.), Developments in Dairy Chemistry, vol. 1, Applied Science Publishers, London, 1982, pp. 61-86.

[21] D.J. McMahon, W.R. McManus, Rethinking casein micelle structure using electron microscopy, Journal of Dairy Science 81 (1998) 2985-2993.

[22] D.S. Horne, Ethanol stability, in: P.F. Fox, P.L.H. McSweeney (Eds.), Advanced Dairy Chemistry Proteins, vol. 1 (Chapter 22), Kluwer Academic, Plenum, New York, 2002. 